\title{
Analytical study of nonlinear phase shift through stimulated Brillouin scattering in single mode fiber with the pump power recycling technique.
}

\begin{abstract}
We present a theoretical study of nonlinear phase shift through stimulated Brillouin scattering in single mode optical fiber. Analytical expressions describing the nonlinear phase shift for the pump and Stokes waves in the pump power recycling technique have been derived. The dependence of the nonlinear phase shift on the optical fiber length, the reflectivity of the optical mirror and the frequency detuning coefficient have been analyzed for different input pump power values. We found that with the recycling pump technique, the nonlinear phase shift due to stimulated Brillouin scattering reduced to less than $0.1 \mathrm{rad}$ for $5 \mathrm{~km}$ optical fiber length and 0.65 reflectivity of the optical mirror, respectively, at an input pump power equal to $30 \mathrm{~mW}$.
\end{abstract}

Keyword: Stimulated Brillouin scattering; Nonlinear phase shift. 